\title{
Influence of cyclic loading on the nutrition of articular cartilage
}

\author{
B P O’Hara, J P G Urban, A Maroudas
}

\begin{abstract}
Articular cartilage is avascular. Nutrients are transported to the cells mainly by diffusion from the synovial fluid. Nutrient transport is also sometimes thought to be assisted by movement of fluid in and out of cartilage in response to cyclic loading of the tissue ('pumping'). The influence of pumping on transport of solutes through cartilage was measured by subjecting plugs of human femoral head cartilage immersed in medium containing radioactive solutes to a simulated walking cycle of $2.8 \mathrm{MPa}$ at $1 \mathrm{~Hz}$. The rate of absorption or desorption of tracers from the cycled plugs was compared with that of unloaded control plugs. For small solutes (urea, $\mathrm{NaI}$ ) fluid transport did not affect the rate of solute transport significantly. Most major nutrients, such as glucose and oxygen, are small solutes and thus nutrition should not be affected by pumping. The rate of desorption of a large solute (serum albumin), however, was increased by $30-100 \%$ in plugs subjected to cyclic loading.
\end{abstract}

Articular cartilage is avascular and its nutrition has been a subject of interest for a number of years. It is accepted that transport of nutrients to the cells takes place mainly from the synovial fluid and that diffusion through the matrix plays a large part. It has also been suggested that the movement of fluid into and out of cartilage aids nutrition and removal of by-products of metabolism by 'pumping' solutes through the matrix. Indeed, inadequate nutrition because of absence of pumping has been suggested as the cause of the detrimental changes found in unexercised cartilage. ${ }^{1}$

That pumping is important for the supply of nutrients of low molecular weight, such as oxygen or glucose, is not supported by any experimental evidence. Qualitative studies showed no difference in the degree of dye penetration into human articular cartilage in the absence or presence of intermittent loading. ${ }^{2}$ In the intervertebral disc, a related tissue, in vivo and in vitro studies found no significant increase in the amount of radioactive tracer sulphate entering the disc as the result of

Bone and Joint Research Unit, London Hospital Medical College, London E1 2AD B P O'Hara

University Laboratory of Physiology Physiology, 1 3PT Oxford OX1

Biomedical Engineering, Israel Institute of Technology, Haifa, Israel

A Maroudas

Correspondence to: Dr Urban.

Accepted for publication 26 June 1989 diffusion coefficient decreases, however, and Thus pumping may assist the movement of high molecular weight substances, such as enzyme inhibitors or hormone carrier-protein complexes, through the matrix. Preliminary results suggest that for serum albumin (mol wt 68000 ) this is indeed the case. ${ }^{6}$

We thus compared the effect of intermittent compression, simulating the walking cycle, on the transport rate of two small solutes (urea and sodium iodide) and one large solute (human serum albumin(HSA)) into human femoral head cartilage. Urea was chosen as it is not sterically excluded by cartilage and has a partition coefficient close to unity $^{7}$; HSA was chosen as the large solute because it is one of the few high molecular weight solutes whose diffusion and partition behaviour in cartilage has been studied; we also examined sodium iodide transport as it is possible experimentally to measure the transport of radioactive iodide and HSA in the same cartilage plug.

\section{Materials and methods}

MATERIALS

Human femoral heads obtained at necropsy were sealed in airtight plastic bags and stored at $-20^{\circ} \mathrm{C}$ until used. Full depth plugs, $9 \mathrm{~mm}$ in diameter, were cored into the underlying bone from areas of the joint where the cartilage surface was intact. The bone was trimmed to $2 \mathrm{~mm}$ thickness.

The medium used was phosphate buffered saline $\mathrm{pH} 7 \cdot 4$, containing $0.01 \%$ sodium azide, protease inhibitors, ${ }^{8}$ and $2 \%$ HSA (grade V, Sigma Chemical Co).

$\left[{ }^{22} \mathrm{Na}\right],\left[{ }^{14} \mathrm{C}\right]$ urea, and $\left[{ }^{125} \mathrm{I}\right]$ iodide were obtained from Amersham International. ${ }^{125}$ I labelled HSA was prepared by the chloramine T method, ${ }^{9}$ and purified as described previously ${ }^{10}$ to separate the free $\left[{ }^{125} I\right]$ iodide from the iodinated protein. As found in earlier studies, ${ }^{10} 11$ however, it was not possible to eliminate all traces of free iodide and the experimental procedure had to be adjusted accordingly.

\section{METHODS}

Cyclic loading

Figure 1 shows the device used, modified from that described by Weightman. ${ }^{12}$ The apparatus consisted of two arms which supported weights. A plunger was attached to the end of each arm and load was transmitted through the plunger to a cartilage plug mounted in a cell. The load on the plug was controlled by adjusting the weight carried by the relevant arm. Experiments were designed so that one cartilage plug was loaded (the experimental plug) and one was not (the control plug). For all tests reported here the load was adjusted so that the experimental plug was compressed by a force of $2.8 \mathrm{MN} / \mathrm{m}^{2}$. The loaded plugs lost fluid and therefore height during the first one to three hours of loading (in preparation) and the initial height of the plunger in contact with the loaded plugs was adjusted to take account of this loss. The height of the control plunger was adjusted so that 


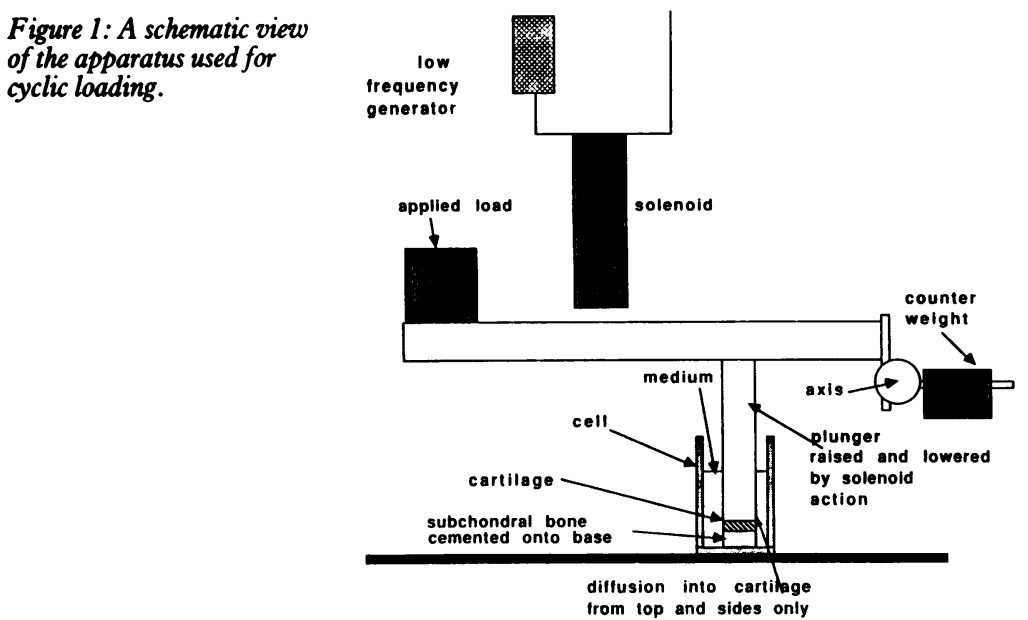

while the plunger came into contact with the surface of the control plug it did not compress it, and the load was counterbalanced so that no force was applied to the control plug. For cyclic loading tests the arms could be lifted away from the plugs by action of a solenoid activated by a $1 \mathrm{~Hz}$ square wave. The timing was adjusted so that the plungers remained in contact with both experimental and control plugs for $38 \%$ of the cycle.

\section{Uptake}

This method was used for measuring the effect of cyclic loading on the rate of uptake of $\left[{ }^{14} \mathrm{C}\right]$ urea into cartilage. Two adjacent plugs were mounted in the cell; one was cyclically compressed and one acted as a control. The loading device was started, and at time zero $3 \mathrm{ml}$ of medium containing 0.004 $\mathrm{mmol} / \mathrm{ml}$ urea and $18.5 \mathrm{kBq} / \mathrm{ml}\left[{ }^{14} \mathrm{C}\right]$ urea was pipetted into the cell. After a suitable time the solution was sucked from the cell as quickly as possible, the device halted, and the cartilage plug blotted. The cartilage plugs were then removed from the holder, sliced from the bone, weighed, and desorbed three times into $3 \mathrm{ml}$ tracer-free medium containing urea. The plugs were dried to constant weight and digested in papain. ${ }^{13}$ The pooled desorption solutions from each plug, the cartilage digests, and an aliquot of the medium were counted in a $\beta$ scintillation counter. In all cases the digest was virtually tracer free. The fractional uptake was calculated as (cpm pooled desorbates $/ \mathrm{g}$ cartilage $) /(\mathrm{cpm} / \mathrm{ml}$ medium). Using this procedure we examined uptake into six pairs of plugs after each of 10, 20, and 60 minutes. The fractional uptake into the loaded plugs was compared with the uptake into the control plugs at each time interval. All experiments were carried out at $4^{\circ} \mathrm{C}$. The results were compared with those calculated for diffusion into the face of a plug 1.6 $\mathrm{mm}$ thick (the average thickness of the plugs used), using a diffusion coefficient of $2.5 \times 10^{-6}$ $\mathrm{cm}^{2} / \mathrm{s}^{14} 15$

Desorption and measurement of desorption rates We chose to measure the effect of loading on desorption rather than on tracer uptake in most tests on large solutes because of complications resulting from the presence of free ${ }^{125} I$ in the medium when testing iodinated proteins. As discussed previously ${ }^{11}$ the partition coefficient of iodide in cartilage is about 0.5 , whereas that of HSA is about $0.01-0.001$ - that is, $50-500$ times less. Thus if cartilage is equilibrated in medium containing ${ }^{125}$ I labelled HSA contaminated by only $0.5 \%$ free iodide the free $\left[{ }^{125} \mathrm{I}\right]$ iodide may account for more than $50 \%$ of the total activity in the cartilage. In the initial stages of equilibration free ${ }^{125}$ I] iodide will account for an even greater fraction of the tracer activity as its diffusion coefficient in cartilage is about 30 times greater than that of HSA. ${ }^{11}$ The rate of ${ }^{125}$ I uptake may be determined by measuring the increase in activity of the cartilage plug with time in a gammacounter. This procedure does not enable the counts from $\left[{ }^{125} I\right]$ iodide to be distinguished from those of ${ }^{125} I$ labelled HSA, however. To determine the activity of each species the tracers must be desorbed into solution and the species separated (by trichloroacetic acid (TCA) precipitation as described); and thus only one time point can be obtained from each plug as was the case with $\left[{ }^{14} \mathrm{C}\right]$ urea.

With urea the molal partition coefficient was almost unity for all plugs, and thus the results from different plugs were pooled. For a large solute such as HSA, however, the partition coefficient decreases exponentially with increase in fixed charge density (a measure of proteoglycan concentration). ${ }^{15}$ Thus the overall partition coefficient of HSA in a plug is very sensitive to the fixed charge density profile; plugs with a relatively large surface zone of low fixed charge density tend to have a much greater overall HSA partition coefficient than thicker plugs with a relatively small surface zone. ${ }^{11}$ Hence the uptake of HSA may vary substantially from plug to plug and the results from different plugs cannot be pooled successfully (figs 3 and 4). Thus it is difficult to determine a time course for HSA uptake. If, however, desorption of ${ }^{125} \mathrm{I}$ from the plug is followed instead, the tracer appearing in the medium can be subjected to TCA precipitation or chromatography ${ }^{10}$ and the two tracer species separated. Thus by sampling the desorbing medium at suitable times the time course of desorption can be followed.

Desorption rates were measured as follows: plugs prepared as for the uptake tests were equilibrated against $3 \mathrm{ml}$ medium containing tracer HSA for 60 hours. At the end of the equilibration period the medium containing tracer was sucked from the holder, the plugs and device were carefully and quickly wiped, and $3 \mathrm{ml}$ of fresh medium containing unlabelled solute-that is, with no radioactive tracer, was added and the oscillating indenter set in motion. At suitable time intervals the desorbing medium was pipetted from the holder and replaced with fresh medium as quickly as possible. The process was repeated until desorption was complete-that is, until no counts could be detected in the medium. The plugs were then removed from the holder, sliced from the bone, and their fixed charge density measured by tracer cation exchange ${ }^{16}$ and their water content from the wet and dry weights. ${ }^{13}$

The total activity of the medium samples and of the original equilibrating solution was measured by a gamma scintillation counter. The activity of HSA in these samples was then determined by subjecting them to a double TCA precipitation ${ }^{10}$ and determining the activity of the precipitates.

The fractional degree of desorption was determined from $(\mathrm{Ni}) / \mathrm{N}$ where $\mathrm{Ni}=\mathrm{ci} \times \mathrm{vi}$, with ci the $\mathrm{cpm} / \mathrm{ml}$ of the medium removed at the ith time interval, vi the volume of the desorbing solution $(3 \mathrm{ml}$ ), and $\mathrm{N}$ the total activity desorbed from the 
plug-that is, $\mathrm{N}$ is summed over all $\mathrm{Ni}$. The partition coefficient of the plug was calculated as $\mathrm{K}=\left(\left(\mathrm{N} /\left(\mathrm{Wo}_{\mathrm{o}}-\mathrm{Wd}\right)\right) / \mathrm{Ns}\right)$ where $\mathrm{N}$ was the total activity of free iodide or HSA desorbed from the plug summed over all the medium samples, Wo was the wet weight of the slice, Wd the dry weight, and Ns the activity of the relevant solute in the original equilibrating solution. Where $\mathrm{K}_{\mathrm{HSA}}$ of the experimental plug differed from that of its paired control by more than $5 \%$ the results were discarded.

Results

Figure 2 shows the fraction of $\left[{ }^{14} \mathrm{C}\right]$ urea absorbed versus time. It can be seen that rate of tracer uptake into the cyclically loaded plugs was very similar to that into the controls. At no time was the difference between the two sets of data significant. Thus pumping does not noticeably assist the movement of urea through cartilage in the configuration tested here. As the rate of diffusion of small metabolites such as glucose, oxygen, and amino acids through cartilage is similar to that of urea $^{15}$ pumping would not be expected to assist movement of these nutrients significantly either.

Figure 3 shows the effect of cyclic loading on the movement of serum albumin and free iodide from the same plug. Cyclic loading had no noticeable effect on the desorption of free iodide. Thus pumping did not influence either the uptake or the desorption of small solutes. Cyclic loading increased the rate of HSA desorption markedly, however.

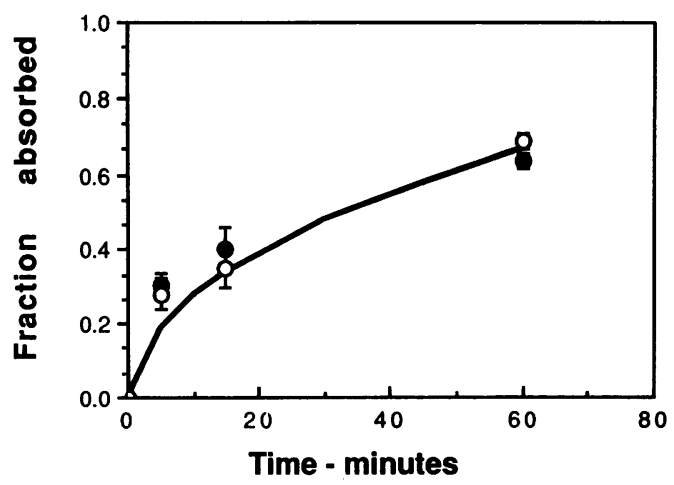

Figure 2: Effect of cyclic loading on uptake of $\left[{ }^{14} C\right]$ urea into cartilage plugs. Each point represents the mean and standard deviation of six independent tests. $\mathrm{O}=$ control plugs; $\mathbf{O}=$ loaded plugs; $-=$ the calculated uptake of urea into the face of a plug $1 \cdot 6 \mathrm{~mm}$ thick.

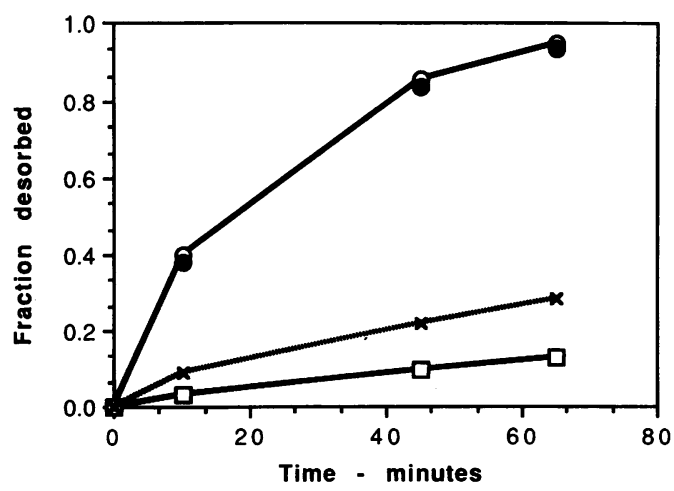

Figure 3: Effect of cyclic loading on the desorption of free Figure 3: Effect of cyclic loading on the desorption of free
12SI and of ${ }^{\prime} S_{\text {I labelled human serum albumin }}(H S A)$ from a cartilage plug. $O=$ free ${ }^{125} I$, loaded plug; $\mathrm{O}=$ free ${ }^{125} I$ control plug; $\times=H S A$ loaded plug; $\square=H S A$ control plug.
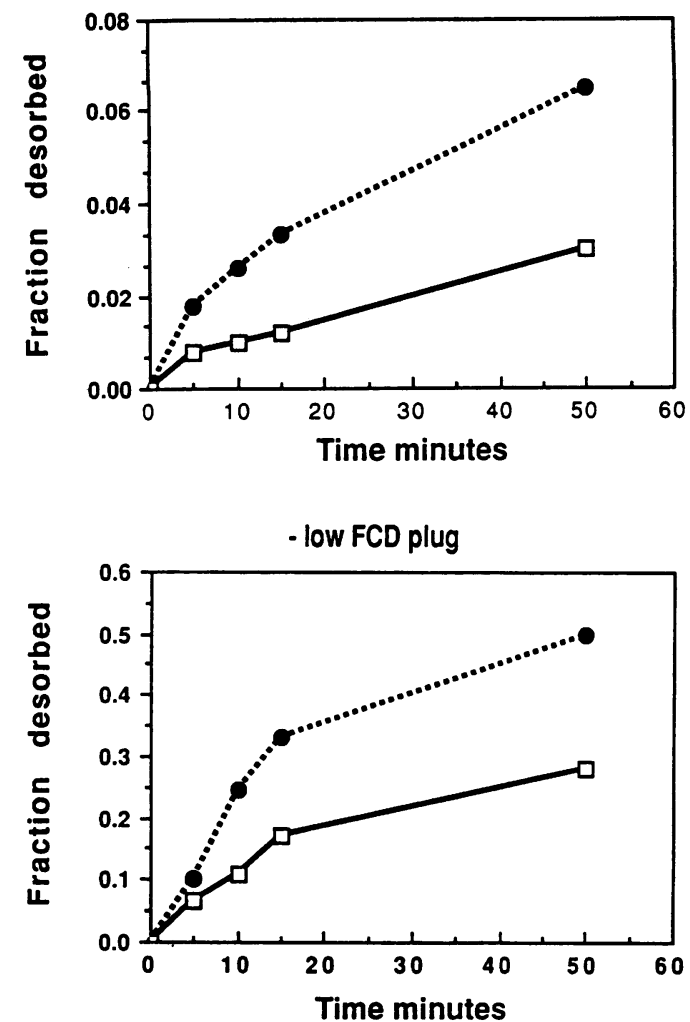

Figure 4: Effect of cyclic loading on the desorption of 125 I labelled human serum albumin from two pairs of plugs, one of high fixed charge density $(F C D)(0 \cdot 17 \mathrm{mE} / \mathrm{g})$ and one of low fixed charge density $(0.09 \mathrm{mE} / \mathrm{g}) . \quad=$ loaded plugs; $\square=$ control plugs.

Figure 4 shows the effect of cyclic loading on the desorption of HSA for two more typical pairs of plugs. Again, in each case, cyclic loading increased the rate of desorption of HSA significantly. Because of variation in plug composition the rate of desorption of HSA varied considerably between plugs for both controls and for cyclically loaded plugs (figs 3 and 4). After 50 minutes' desorption the fraction of HSA desorbed from the controls ranged from 0.05 to 0.27 , and from the loaded plugs from 0.07 to 0.5 ; in general the rate of desorption was fastest from plugs of low fixed charge density, probably because fluid permeability and HSA diffusivity is highest in these plugs. ${ }^{11}$

We have not attempted to compare these results in detail with those expected from theory. Even for the control plugs the geometry of the system was not simple as both the sides and the cartilage surface were exposed to solution; with a cartilage thickness of 1.3 to $2.0 \mathrm{~mm}, 20-30 \%$ of the exposed area came from the sides. In addition, during loading the plunger cut off the surface from contact with solution for part of the cycle. In the loaded plugs, deformation during loading, which altered the diffusion distance, further complicated any analysis. The fluid flow patterns produced during loading were highly non-uniform. During cyclic loading at $1 \mathrm{~Hz}$ radial flow probably only occurred in the outer periphery of the plug, ${ }^{17}$ and thus it is only in this region that diffusion will compete with fluid flow. Although the loading pattern leads to an overall gradual fluid loss (in preparation), convective gradients induced at each loading cycle probably do not penetrate throughout the plug. If the complications discussed above are ignored, however, the rate of uptake of urea is not much greater than that predicted for diffusion 
(fig 3) into the face alone, confirming that here diffusion is the major mechanism for transport of small solutes through the cartilage plug.

\section{Discussion}

Solutes may move through the cartilage matrix both by diffusion, and by transportation with fluid which moves through the tissue when it is loaded. The relative influence of fluid flow on solute transport will depend on the ratio of $\mathrm{D}$, the solute diffusion coefficient, to $\mathrm{D}(\mathrm{H})$, the fluid transport coefficient. The influence of fluid flow on solute transport will thus depend on solute size as in general the value of the diffusion coefficient, D, decreases as solute size increases. Whether convective flow influences solute transport will also depend on tissue composition and on how the tissue is loaded because these factors influence the value of $\mathrm{D}(\mathrm{H})$. The magnitude of $\mathrm{D}(\mathrm{H})$ is greatest-that is, fluid flow is fastest, where the pressure gradient is high and where the cartilage samples are degenerate and have lost proteoglycans. ${ }^{18}$ Solute size also influences convective drag since the 'effective' $\mathrm{D}(\mathrm{H})$ falls by up to $50 \%$ as solute size increases because of viscous and fractional effects. ${ }^{5}$ The direction of fluid flow may also be important as under some conditions fluid movement may oppose solute transport rather than assist it.

We have shown here that fluid movement does not contribute significantly to the rate of movement of small solutes through the cartilage plugs used either when fluid movement opposes transport (fig 2) or when it assists it (fig 3). This is not surprising when the relative coefficients for fluid movement and diffusion are compared. The diffusion coefficients, $D$, for small solutes in cartilage (oxygen, glucose, lactate, urea, inorganic ions) are in the range $2-6 \times 10^{-6} \mathrm{~cm}^{2} / \mathrm{s},{ }^{15}$ whereas the fluid transport coefficient, $\mathrm{D}(\mathrm{H}),{ }^{19}$ for physiological loading conditions lies between $0.2 \times 10^{-7}$ and $2 \times 10^{-7} \mathrm{~cm}^{2} / \mathrm{s}$, and is thus $10-100$ times smaller than D. ${ }^{18}$ For large solutes such as HSA, however, the value of $D$ is about $2 \times 10^{-7} \mathrm{~cm}^{2} / \mathrm{s} .{ }^{15}$ As it is of similar magnitude to $D(H)$, even allowing for viscous and fractional retardation, it is not surprising that fluid movement may effect the transport of such solutes noticeably.

Hence cyclic loading may influence the rate at which large solutes such as growth factors, hormones, enzymes and their inhibitors, and cytokines reach the cells. Such solutes may markedly influence cell metabolism. Pumping may also influence the rate at which newly synthesised matrix macromolecules themselves move through the matrix, and might influence the rate of loss of matrix breakdown products into the synovial fluid.

Although our results indicate that loading does not influence the movement of small nutrients through the matrix itself, loading may nonetheless be important for nutrition in other ways. Nutrients move into articular cartilage mainly from the synovial fluid; loading can influence the production of synovial fluid ${ }^{20}$ and also help distribute it across the joint surface. ${ }^{21}$ Without movement and loading, pools of stag- nant synovial fluid may be depleted of their supply of nutrients, and may accumulate acidic wastes such as lactate and $\mathrm{CO}_{2}{ }^{1}$ In this way lack of movement may cause inadequate 'nutrition' of cartilage.

This work was supported by the Wellcome Trust and the Arthritis and Rheumatism Research Council.

1 McKibbin B, Maroudas A. Nutrition and metabolism. In Freeman M A R, ed. Adult articular cartilage. 2nd ed. London: Pitman Medical, 1979: 461-86.

2 Maroudas A, Bullough P G, Swanson S A V, Freeman M A R. The permeability of articular cartilage. $\mathcal{F}$ Bone foint Surg [Br] 1968; 50: 166-77.

3 Urban J P G, Holm S, Maroudas A, Nachemson A. Nutrition of the intervertebral disc: effect of fluid flow on solute transport Clin Orthop 1982; 170; 296-306.

$4 \mathrm{Katz}$ M, Hargens A R, Garfin S R. Intervertebral disc nutrition diffusion versus convection. Clin Orthop 1986; 210: 243-5.

5 Curry F-R E. Mechanics and thermodynamics of transcapillary exchange. In: Renkin E M, Michel C C, eds. Handbook of physiology 2-cardiovascular system IV. Bethesda, Marlyand: American Physiological Society, 1984; 337

6 Tomlinson N, Maroudas A. The effect of cyclic and continuous compression on the penetration of large molecules into articular cartilage. $\mathcal{F}$ Bone foint Surg $[\mathrm{Br}] 1980$ 62: 251 .

7 Maroudas A. Distribution and diffusion of solutes in articular cartilage. Biophys 7 1970; 10: 365-79.

8 Tyler J A. Chondrocyte-mediated depletion of articular cartilage proteoglycans in vitro. Biochem 7 1985; 225: cartilage

9 Hunter W M, Greenwood F C. Preparation of iodine-131 labelled human growth hormone of high specific activity. Nature 1962; 194: 495-6.

10 Snowden J, Maroudas A. The distribution of serum albumin in human normal and degenerate cartilage. Biochim Biophys Acta 1976; 428: 726-33.

11 Maroudas A. Transport of solutes through articular cartilage: permeability to large molecules. $\mathcal{F}$ Anat $1976 ; 122: 335-47$.

12 Weightman B O. Tensile fatigue of human articular cartilage. 7 Biomech 1976; 9: 193-200.

13 Venn M F, Maroudas A. Chemical composition and swelling in normal and osteoarthritic femoral head cartilage. I. Chemical composition. Ann Rheum Dis 1977; 36: $399-406$.

14 Crank J. The mathematics of diffusion. 2nd ed. Oxford: Oxford University Press, 1975.

15 Maroudas A. Physicochemical properties of articular cartilage. In: Freeman M A R, ed. Adult articular cartilage. 2 nd ed. London: Pitman Medical, 1979: 215-90.

16 Maroudas A, Thomas H. A simple physicochemical micromethod for determining fixed anionic groups in connective tissue. Biochim Biophys Acta 1971; 215: 214-6.

17 Armstrong C G, Lai W M, Mow V C. An analysis of the unconfined compression of articular cartilage. $\mathcal{f}$ Biomech Eng 1984; 106: 165-73.

18 Urban J, Maroudas A. Fluid flow in the intervertebral disc in relation to creep. Proceedings of the Society of Mechanical Engineers, 1980: C88.

19 Fatt I Goldstick $\mathrm{T} F$. Dynamics of water transport in swelling membranes. Fournal of Colloid Science 1965; 20 : 962-89.

20 Levick J R. Synovial fluid dynamics: the regulation of volume and pressure. In: Maroudas A, Holborow E J, eds. Studies in joint diseases. Vol 2. London: Pitman Medical, 1983: $153-240$.

21 Levick J R. Blood flow and mass transport in synovial joints. In: Renkin E M, Michel C C, eds. Handbook of physiolog 2-the cardiovascular system part IV. Bethesda, Maryland: American Physiological Society, 1984: 917-48. 